\title{
Application of bias correction methods to improve the accuracy of quantitative radar rainfall in Korea
}

\author{
J.-K. Lee ${ }^{1}$, J.-H. Kim² ${ }^{2}$ and M.-K. Suk ${ }^{2}$ \\ ${ }^{1}$ Innovation Center for Engineering Education, Daejin University, \\ Pocheon-si, Gyeonggi-do, Korea \\ ${ }^{2}$ Weather Radar Center, Korea Meteorological Administration, Seoul, Korea \\ Received: 18 March 2015 - Accepted: 18 March 2015 - Published: 22 April 2015 \\ Correspondence to: J.-K. Lee (myroom1@ daejin.ac.kr) \\ Published by Copernicus Publications on behalf of the European Geosciences Union.
}

4011

\begin{abstract}
There are many potential sources of bias in the radar rainfall estimation process. This study classified the biases from the rainfall estimation process into the reflectivity measurement bias and QPE model bias and also conducted the bias correction methods to 5 improve the accuracy of the Radar-AWS Rainrate (RAR) calculation system operated by the Korea Meteorological Administration (KMA). For the $Z$ bias correction, this study utilized the bias correction algorithm for the reflectivity. The concept of this algorithm is that the reflectivity of target single-pol radars is corrected based on the reference dual-pol radar corrected in the hardware and software bias. This study, and then, dealt 10 with two post-process methods, the Mean Field Bias Correction (MFBC) method and the Local Gauge Correction method (LGC), to correct rainfall-bias. The $Z$ bias and rainfall-bias correction methods were applied to the RAR system. The accuracy of the RAR system improved after correcting $Z$ bias. For rainfall types, although the accuracy of Changma front and local torrential cases was slightly improved without the $Z$ bias 15 correction, especially, the accuracy of typhoon cases got worse than existing results. As a result of the rainfall-bias correction, the accuracy of the RAR system performed $Z$ bias_LGC was especially superior to the MFBC method because the different rainfall biases were applied to each grid rainfall amount in the LGC method. For rainfall types, Results of the $Z$ bias_LGC showed that rainfall estimates for all types was more 20 accurate than only the $Z$ bias and, especially, outcomes in typhoon cases was vastly superior to the others.
\end{abstract}

\section{Introduction}

Weather radars can provide rainfall estimates over the Korean Peninsula and near seas with high spatial (minimum $0.125 \mathrm{~km}$ ) and temporal resolutions $(2.5 \mathrm{~min})$, especially, and play an important role in predicting and monitoring severe weather conditions. However, several sources of bias are involved in the process of calculating quantita- 
tive radar-based rainfall estimates. It is well acknowledged that radar data are affected by both systematic bias (due to reflectivity measurements (included in hardware errors, signal processing, and quality controls), parameter estimation of the $Z-R$ relationship, and quantitative precipitation estimation model structures) and random error 5 (Huff, 1970; Woodely et al., 1957; Wilson and Brandes, 1979; Austin, 1987; Campos and Zawadzki, 2000; Krajewski and Smith, 2002) because one of major reasons is that weather radars do not measure rainfall amounts directly but estimate the rainfall in the process of converting $Z\left(\mathrm{~mm} \mathrm{~m}^{-3}\right)$ into $R\left(\mathrm{~mm} \mathrm{~h}^{-1}\right)$ using the empirical relationship in the form of the $Z-R$ relationship. Related to systematic bias, a considerable number

10 of studies have been conducted to correct the reflectivity measurement bias which includes temporal and spatial sampling bias, ground and sea clutter, beam-blockage and attenuation, electrical calibration, and quantification of reflectivity bias (Chumchean et al., 2006). Jordan et al. (2000) evaluated the errors which arise in radar estimates of rainfall as a result of temporal sampling, spatial averaging, measuring the field at some

15 distance above the ground, and recording the reflectivity data with a limited radiometric resolution. Germann et al. (2006) modified the ground clutter algorithm and reduced the amount of residual non-meteorological signals in a mountainous region, the Alps, to improve the precipitation estimation. Villarini and Krajewski (2008) investigated the spatial sampling errors in radar observations which affect the sensitivity of the models

20 and determined that these errors were related to the approximation of an areal estimate by a using a point measurement. Similarly, converting a measured reflectivity to rainfall amount using artificial relationships or models is one of the major sources of bias. To overcome these limitations, gauge adjustment methods were applied to correct misestimated precipitation in numerous existing studies. Sinclair and Pegram (2005) described a merging technique and presented an application of it to a simulated rainfall field. The proposed merging technique based on Conditional Merging (CM) (Ehret, 2002) made use of a Kriging method to reduce the bias while retaining spatial detail from the radar but keeping the spatial variability observed by the radar. Morin and Gagella (2007) compared three radar-gauge adjustment methods, a one-coefficient bulk adjustment,

4013

a Weighted Regression (WR), and a Weighted Multiple Regression (WMR), for the radar-based quantitative precipitation estimation over Mediterranean and dry climate regimes. They concluded the WR and WMR adjustment methods were useful for calculating rain depth estimates, with some limitations. Goudenhoofdt and Delobbe (2009)

5 dealt with several radar-gauge merging methods considering the gauge network densities and compared their precipitation estimates accuracy. The analysis revealed that the simple methods reduced relatively the bias of radar estimation and the geostatistical merging methods resulted in the better performance reflecting the gauge network densities.

10 In a series of procedures which estimate the quantitative rainfalls derived from radar information, the present paper focuses on correcting the measurement bias and bias in the QPE model. The measurement bias is defined as the reflectivity measurement bias (hereafter $Z$ bias) which was reflected in all bias which occurred while using weather radar to detect precipitation. The bias in the QPE model (hereafter QPE mode bias)

15 is defined as the estimated rainfall-bias which included the bias due to the parameters of the $Z-R$ relationship, the parameters of the QPE model, and the QPE model structure. Section 2 describes the correction methods of the $Z$ bias and rainfall-bias and the QPE model used in this paper. Section 3 gives results for rainfall estimations using the correction methods and describes the effect of the $Z$ bias and rainfall-bias correc-

20 tion methods. Finally, Sect. 4 summarizes the results and provides some concluding remarks.

\section{Data and methodology}

\subsection{Radar dataset and rainfall cases}

In this study, the performance of the bias correction methods has been evaluated by 25 comparing the observed rainfall data from rain gauges operated by the KMA (Korea Meteorological Administration). Observed rainfall data were collected from 642 rain 
gauges (321 rain gauges for the calibration and 321 rain gauges for the validation) located in the Korean Peninsula. The Bislsan $S$ band dual-polarimetric radar which was installed and operated by the Ministry of Land, Infrastructure and Transport (MLIT) beginning in 2009 was selected for the absolute reference radar to estimate $Z$ bias (de-

5 scribed in Sect. 2.2). Horizontal and vertical reflectivity $\left(Z_{\mathrm{H}}\right.$ and $\left.Z_{\mathrm{V}}\right)$, differential reflectivity $\left(Z_{\mathrm{DR}}\right)$, differential phase $\left(\Phi_{\mathrm{DP}}\right)$, specific differential phase $\left(K_{\mathrm{DP}}\right)$, correlation coefficient $\left(\rho_{\mathrm{HV}}\right)$, and spectrum width (SW) with a scan range of the maximum $150 \mathrm{~km}$ and a gate size of $0.125 \mathrm{~km}$. The scan strategy has 6 elevation angles with a 2.5 min update cycle. The target radars which required $Z$ bias correction were 11 single-polarimeric

10 radars (Baegnyeondo, Kwanaksan, Oseonsan, Jindo, Gosan, Seongsan, Gudeoksan, Myeonbongsan, Gangneung, Gwnagdeoksan, Incheon) with a scan range of the maximum $200 \mathrm{~km}$ ( $C$ band) and $240 \mathrm{~km}$ ( $S$ band) and a gate size of $0.250 \mathrm{~km}$ operated by the KMA in Fig. 1. Table 1a shows the rainfall event periods of the radars and raingauges used for estimating $Z$ bias and Table $1 \mathrm{~b}$ shows 18 rainfall cases in the summer 15 season used for the verification of the $Z$ and rainfall-bias correction methods.

\subsection{Quantitative precipitation estimation model}

This paper has utilized the Radar-AWS Rainrate (RAR) calculation system (hereafter called the RAR system) for the QPE model. The RAR system which was developed by KMA in 2006 is operated on site, based on 11 single-polarimetric radars. The RAR

20 system produces the merged rainfall field for the Korean Peninsula through a series of steps (production of the radar reflectivity field, calculation of AWS rainfalls, derivation of the $Z-R$ relationship, etc.) (refer to Fig. 2).

The RAR system estimates parameters of the $Z-R$ relationship in real-time for realtime rainfall estimates (Weather Radar Center, 2011). The RAR system utilizes $10 \mathrm{~min}$

25 reflectivity and AWS rainfall in the Window Probability Matching Method (WPMM) (Rosenfeld et al., 1993) to estimate rainfalls in each radar site and merged rainfalls of radar sites for producing composite rainfall fields. Used reflectivity which are quality controlled (removal of non-meteorological echoes) are averaged on $3 \times 3$ pixels with 4015

a certain AWS as the center are used. The WPMM method reproduces the probability density functions (pdfs) of ground rainfall from AWSs and radar reflectivity and determines the $Z-R$ relationship using these pdfs (refer to Eqs. 1 and 2) (Rosenfeld et al., 1993).

$$
\begin{aligned}
& \int_{0}^{\infty} f\left(Z_{\mathrm{e}}\right) P_{\mathrm{c}}\left(Z_{\mathrm{e}}\right) \mathrm{d} Z_{\mathrm{e}}=\int_{0}^{\infty} R P_{\mathrm{c}}(R) \mathrm{d} R, \\
& P_{\mathrm{c}}(R)=P\left(R \mid R>R_{T}\right), \quad P_{\mathrm{c}}\left(Z_{\mathrm{e}}\right)=P\left(Z_{\mathrm{e}} \mid Z_{\mathrm{e}}>Z_{\mathrm{e} T}\right),
\end{aligned}
$$

where $Z_{\mathrm{e}}$ is radar reflectivity $(\mathrm{dBZ}), P_{\mathrm{c}}$ is the conditional probability function, $R$ is rainfall $\left(\mathrm{mm} \mathrm{h}^{-1}\right)$, and $T$ is threshold. The conditional probability functions in Eq. (1) are derived from Eq. (2) and thresholds of rainfall and radar reflectivity are $0.1 \mathrm{~mm} \mathrm{~h}^{-1}$ and

$1010 \mathrm{dBZ}$. Parameters of the $Z-R$ relationship have been estimated using radar reflectivity and AWS rainfalls from $1 \mathrm{~h}$ ago with the least square fit of power law. The number of radar reflectivity and AWS rainfalls over a certain threshold are required to estimate parameters accurately. If there is not enough data, estimated rainfalls from that $Z-R$ relationship are inaccurate. To overcome this limitation, if the number of available AWSs

15 is more than $30 \%$ of those available in each radar site, the parameters of the $Z-R$ relationship can be estimated. If less than $30 \%, Z=200 R^{1.6}$ (Marshall and Palmer, 1948 ) is applied for rainfall estimates (Korea Meteorological Administration, 2012b).

Secondly, the composite rainfall field for the whole country may be produced using each radar rainfall estimate. However, appropriate merging methods must be con-

20 ducted because the scan ranges of the radar sites overlap. In this paper, four merging methods are available as follows: (i) the maximum value method, which selects the maximum value among radar rainfalls in the overlapping area, (ii) the average value method, which averages all radar rainfalls, (iii) the minimum value method, which selects the minimum value among radar rainfalls, and (iv) the distance weighting method,

25 which assigns weights to radar rainfalls according to their distance from a center of a radar to a radar pixel. Because the maximum value method is applied to merge 
radar rainfalls by the KMA (Korea Meteorological Administration, 2012b), the identical method is also utilized in this paper.

\subsection{Bias correction methods}

\subsubsection{Reflectivity measurement bias correction method}

5 Weather radars continuously carry out measurement cycles which include sending signals into the atmosphere and receiving and analyzing return signals for meteorological observation. The measurement of reflectivity itself suffers from hardware malfunctions (e.g. electronic miscalibration, signal misprocessing) and radar characteristics (e.g. attenuation, beam blockage). When converting radar reflectivity into rainrates $(Z-R$ re-

10 lationship), contaminated reflectivity (contained $Z$ bias) leads to additional bias which can lower the accuracy of rainfall estimation. To estimate the $Z$ bias of target weather radars, a reference weather radar which has been absolutely corrected is required. The $Z$ bias is defined as the difference between the measured reflectivity of reference radar and the target radar under the same spatial and temporal conditions (Weather Radar

15 Center, 2012). The procedure of estimating $Z$ bias is described as follows.

\section{Calibration of the reference weather radar}

This paper selected a Bislsan $S$ band dual-polarimeric radar (hereafter Bislsan dual-pol radar) which can be self-calibrated and is more accurate than the reference weather radar. To calibrate the Bislsan dual-pol radar, a self-consistency constraint method using the relationship between the reflectivity $(Z)$ depended by the radar beam power and the specific differential phase $\left(K_{\mathrm{DP}}\right)$ affected by only particle size or concentration, not the radar beam power was utilized. The procedure of the self-consistency constraint method is as follows (Weather Radar Center, 2012):

i. Derive the $Z_{\mathrm{H}}-K_{\mathrm{DP}}$ relationship theoretically from the Drop Size Distributions (DSDs).

4017

ii. Calculate $K_{\mathrm{DP}}$ for each radar pixel from observed $Z_{\mathrm{H}}$ using the derived $Z_{\mathrm{H}}-K_{\mathrm{DP}}$ relationship and $\Phi_{\mathrm{DP}}$ as integrating calculated $K_{\mathrm{DP}}$ along each radial.

iii. Calculate the difference angle $(\theta)$ using a scatter plot between the calculated $\Phi_{\mathrm{DP}}$ from (ii) and observed from the Bislsan dual-pol radar and calculate $Z$ bias $(\varepsilon)$ by inputting the difference angle $(\theta)$ into Eqs. (3) and (4) (Lee et al., 2006) (refer to Fig. 3).

$\tan \theta=\frac{\sum_{i=1}^{n}\left(\Phi_{\mathrm{DP} \_c a l}-\Phi_{\mathrm{DP} \_ \text {obs }}\right)}{\sum_{i=1}^{n} \Phi_{\mathrm{DP} \_ \text {obs }}^{2}}$,

$\varepsilon(\mathrm{dB})=10 b \log (\tan \theta)$,

where $\Phi_{D_{\text {P_cal }}}$ is theoretical $\Phi_{\mathrm{DP}}$ from DSDs, $\Phi_{\mathrm{DP} \_ \text {cal }}$ is observed $\Phi_{\mathrm{DP}}$ from the dual10 pol radar, $\theta$ is the difference angle, $b$ is the empirical constant, and $\varepsilon$ is the estimated $Z$ bias.

\section{Calculation of $Z$ bias for the target weather radars}

After completed to calibrate the Bislsan dual-pol radar for $Z$ bias, target single-pol radars which are located adjacent to the reference radar were calibrated according to 15 the reflectivity of the reference radar. The procedure for calculating the $Z$ bias of the target radars is as follows (Korea Meteorological Administration, 2011):

i. Remove the beam-blockage area using beam-blockage information (penetration ratio more than $90 \%$ ).

ii. Reflect the accumulated attenuation effects due to rainfall in the observed reflectivity (attenuation ratio less than $10 \%$ ). 
iii. Generate the 3-dimensional CAPPI for the reflectivity.

iv. Set up equidistant pairs between the reference and target radars within $200 \mathrm{~km}$ from the center of the reference radar. (However, when a Bislsan dual-pol radar was a reference radar, the distance was within $100 \mathrm{~km}$.)

v. Compare the reflectivity of the reference and target radars within a $\pm 5 \mathrm{~km}$ reflectivity overlap area.

vi. Calculate the reflectivity differences at intervals of $0.5 \mathrm{~km}$ from $1.5 \sim 3.5 \mathrm{~km}$ altitude in consideration of the ground clutter and bright band and average the reflectivity differences for the $Z$ bias of the target radar.

Figure 4 shows the concept of $Z$ bias for target radar which has been calculated from reflectivity differences in the overlap area between the reference and target radars. After completed to calibrate the target radar\#1 for the $Z$ bias, the target radar\# 1 is the reference radar for the target radar\#2 adjacent to the target radar\#1. The procedure mentioned above is equally applied for the target radar\# 1 and \#2 to calculate the $Z$ bias 15 of target radar\#2.

\subsubsection{Rainfall bias correction method}

Estimated rainfall based on radars has the QPE model bias (parameters of $Z-R$ relationship, parameters of QPE model, QPE model structures, etc.) even if calibrated reflectivity is input into the QPE model. In this paper, the Mean Field Bias Correction (MFBC) method and Local Gauge Correction (LGC) method have been applied to outcomes from the QPE model for correcting the rainfall-bias.

\section{Mean field bias correction method}

The fundamental concept of the MFBC method is that the bias correct factor $(G / R$ ratio factor) is calculated using the ratio of the spatial average (mean) between rainfalls 4019

estimated from radars and observed rainfall at a corresponding field (or point, pixel). Then corrected rainfall is calculated by multiplying the $G / R$ ratio factor and radar rainfall estimates. The equation of the MFBC method is as follows:

$G / R$ ratio factor $=\sum_{i=1}^{n} G_{i} / \sum_{i=1}^{n} R_{i}$,

5 where $G_{i}$ is rainfall of $i$ th rain gauge, $R_{i}$ is radar rainfall estimates of $i$ th point (or pixel), $n$ is the total number of the ground rain gauge. In the case of utilizing the MFBC method in a certain area (or for a certain period), the identical $G / R$ ratio factor is uniformly applied to radar rainfall estimates all over the area.

\section{Local Gauge Correction method}

${ }_{10}$ This study dealt with the Local Gauge Correction (LGC) method which has been employed in the NMQ (National Mosaic and QPE) of the NOAA (National Oceanic and Atmospheric Administration) NSSL (National Severe Storms Laboratory) (Zhang et al., 2011). The LGC method which assigns the weights to bias between ground rainfall detected by Automatic Weather Stations (AWSs) and radar rainfall estimates is the modified version of the Inverse Distance Weighting (IDW) method. The LGC method is able to correct the rainfall cases which occur locally by modifying rainfall estimates in each pixel. The procedure of the LGC method is as follows (refer to Fig. 5):

This paper defined that $r_{\mathrm{LGC}, i}$ is the corrected rainfall estimates in a certain point $i$, $r_{i}$ is radar rainfall estimates in a certain radar pixel $i, R_{\mathrm{e}, i}$ is expected error estimates. 20 This relationship is expressed as following equation:

STEP 1: $r_{\mathrm{LGC}_{, i}}=r_{i}-R_{\mathrm{e}, i}=r_{\mathrm{LGC}, i}(b, D)$,

where $D$ is scan range, $b$ is the weight of variable $d, d$ is the distance between AWSs and pixels in radars. Estimated weights by Eq. (7) are applied to Eq. (6) (Zhang et al., 
2011).

$R_{\mathrm{e}, i}=\sum_{j=1}^{m} e_{j} w_{j} / \sum_{j=1}^{m} w_{j}$

If general, $w_{j}=1 / d_{j}^{b}$ (if $d_{j} \leq D$ ) or 0 (if $d_{j}>D$ ).

If the number of AWS in region are sparse,

${ }_{5} \quad \alpha=\sum_{j=1}^{m} \exp \left[-d_{j}^{2} /(D / 2)^{2}\right]$,

$w_{j}=\alpha \times 1 / d_{j}^{b}$ (if $\left.d_{j} \leq D\right)$ or 0 (if $d_{j}>D$ ),

where $e_{j}$ is error between rainfalls observed from AWSs $\left(g_{j}\right)$ and radar rainfall estimates $\left(r_{j}\right), w$ is the weight of error $\left(=r_{j}-g_{j}\right), j$ is $j$ t AWS, $m$ is the number of AWSs within the radar scan range, $\alpha$ is the impact factor. If the $\alpha$ is more than one, the number 10 of AWSs is enough for the rainfall-bias correction. Otherwise, less than one, if the number of AWSs is sparse (the $\alpha$ is less than one), revised weights have been calculated by multiplying $\alpha$ and original weights.

$E_{i}$ is defined as the difference between $r_{\mathrm{LGC}}$ from STEP 1 and ground rainfall, $g_{i}$, and depends on $b$ and $D$.

15 STEP 2: $E_{i}=r_{\mathrm{LGC}}-g_{i}=E_{i}(b, D)$

Mean Square Error (MSE) for $E_{i}$ is expressed as Eq. (10) and also depends on parameter $b$ and $D$. Parameters of the LGC method ( $b$ and $D$ ) have been determined using the stepwise method for minimizing the MSE value and applied to Eq. (8) to calculate radar rainfall estimates, $r_{\mathrm{LGC}}$.

20 STEP 3: MSE $=\sum_{i=1}^{n} E_{i}^{2} / n=\operatorname{MSE}(b, D)$

4021

This paper has assumed that the scan range of radars $(D)$ is the maximum range $240 \mathrm{~km}$ used by all AWSs on the Korean Peninsula. Although it takes a long time to carry out the LGC algorithm under this assumption, it is considered appropriate to verify the improvement of radar rainfall estimates using the LGC method.

5 In sequence, because the LGC method is highly dependent on the number of AWSs which are available and accurate, the quality control algorithm for AWSs has been conducted to remove lower-quality AWSs which have larger expected errors than others. The conditions of quality control are as follows: (i) in a certain AWS, if the number of pixels which have $D_{\mathrm{R}, \mathrm{E}}$ less than $5 \mathrm{~mm}$ are less than $25 \%$ of the whole pixels, a certain

10 AWS is designated as an "abnormal AWS" and removed. $D_{\mathrm{R}, \mathrm{E}}$ are the differences between $R_{\mathrm{e}, i}$ and $E_{i}$ within $10 \mathrm{~km}$ radius from the center of a certain AWS. (ii) The LGC method has been conducted until the number of available AWSs was more than $90 \%$ of all the filtered AWSs. If this procedure is stopped, calculated $r_{\mathrm{LGC}}$ at the present stage is used for corrected rainfall estimates. (iii) The procedure of the LGC method is finally

15 finished after repeating the routine above approximately four times. Furthermore, if the ratio of abnormal AWSs is more than $7 \%$, the procedure of the LGC method is also finished (Korea Meteorological Administration, 2012). Thresholds were decided using the stepwise method and are appropriate for the LGC method applied to the RAR calculation system. However the thresholds are somewhat subjective, it is considered that 20 future studies should deal with this limitation.

\section{Application and results}

\subsection{Application of the reflectivity measurement bias correction method}

In Sect. 2.21, the reflectivity measurement bias ( $Z$ bias) for the Bislsan dual-pol radar have been estimated using the self-consistency constraint method using the relationship between reflectivity $(Z)$ and specific differential phase $\left(K_{\mathrm{DP}}\right)$ during the calibration period. The $Z$ bias of the Bislsan dual-pol radar was estimated as $-2.61 \mathrm{~dB}$ with the re- 
sult that the calculated $\tan \theta$ which was $0.58^{\circ}$ from Eq. (1) was inputted into Eq. (4). The Bislsan dual-pol radar was self-calibrated using its $Z$ bias. For estimating the $Z$ bias of target radars, first of all, pairs between the reference radar and target radar were set up (refer to Table 2). Then averaged $Z$ biases of the 11 single-pol radars operated 5 by the KMA as target radars were estimated sequentially from the beginning, using the Bislsan dual-pol radar as the reference radar (refer to Fig. 6 and Table 3). The $Z$ biases of the BRI and JNI sites were $-7.87 \mathrm{~dB}$ (the largest) and $-1.16 \mathrm{~dB}$ (the smallest) and $Z$ bias on average was $-4.52 \mathrm{~dB}$. Especially, radar rainfall estimates were underestimated due to the fact that all of the $Z$ biases had negative values.

To verify the improvement of radar rainfall estimates, the RAR system which reflected the $Z$ biases of all the radar sites were conducted to calculate rainfall estimates of 18 cases in the summer season. In Fig. 7, after applying $Z$ biases to the RAR system, the accuracy of rainfall estimates improved in the Root Mean Square Error (RMSE) and correlation coefficient, which ranged from $7.37 \mathrm{~mm} \mathrm{~h}^{-1}$ and 0.83 and $7.21 \mathrm{~mm} \mathrm{~h}^{-1}$ and

150.84 on average, respectively. As a result of each rainfall type, in RMSE, the accuracy of rainfall estimates in Changma front cases was improved from 7.43 to $7.36 \mathrm{~mm} \mathrm{~h}^{-1}$ and the accuracy of local torrential rainfall cases $\left(7.43 \mathrm{~mm} \mathrm{~h}^{-1}\right)$ was similar to results without the application of $Z$ bias $\left(7.36 \mathrm{~mm} \mathrm{~h}^{-1}\right)$. Especially, the accuracy of typhoon cases deteriorated compared to existing results (from 9.08 to $11.04 \mathrm{~mm} \mathrm{~h}^{-1}$ ). This was due to

20 the application of $Z$ biases to each radar site in the RAR system, which has increased the rainfall estimates in whole country. The accuracy of Changma front cases which occur nationwide was improved. However, because cases of local torrential rainfalls and typhoons occurred locally, the accuracy of these cases was negatively impacted. In Fig. 8 in Case 12 at 15:00 LST on 10 August in 2012, Fig. 8a shows the image be25 fore the application of $Z$ bias and Fig. 8b shows the image after the $Z$ bias correction. Rainfall estimates in black dash circles on the partial magnification image in Fig. $8 \mathrm{~b}$ are stronger than Fig. $8 \mathrm{a}$, relatively since the rainfall estimates were increased by the $Z$ bias correction. It is proved that the $Z$ bias correction proposed by this paper has improved the accuracy of rainfall amounts in the RAR system.

4023

\subsection{Application of the QPE model bias correction methods}

Since the rainfall estimates in the RAR system were improved by the $Z$ bias correction in Sect. 3.1, the QPE model bias (rainfall-bias) correction was conducted after the $Z$ bias correction. To verify the improvement of the radar rainfall amounts estimated

5 by the QPE model bias correction, the RAR system with rainfall-bias correction was conducted for 18 summer season cases over the verification period. This paper defined that results with only the $Z$ bias correction were identified as " $Z$ bias", results with the $Z$ bias correction and MFBC method were identified as " $Z$ bias_MFBC", and results with the $Z$ bias correction and LGC method were identified as " $Z$ bias_LGC".

As a result of the rainfall-bias correction methods, Table 4 shows the accuracy of rainfall estimates for each rainfall-bias method and for each rainfall type. In Table 4a, Mean Absolute Error (MAE) of the $Z$ bias, $Z$ bias_MFBC, and $Z$ bias_LGC were 3.65, 3.37, and $2.19 \mathrm{~mm} \mathrm{~h}^{-1}$, respectively. Among them, the accuracy of the $Z$ bias_LGC was superior to the others. In RMSE, the accuracy of rainfall amounts of the RAR 15 system was improved by about $7.4 \%$ (from 7.21 to $6.68 \mathrm{~mm} \mathrm{~h}^{-1}$ ) in the $Z$ bias_MFBC and $63.7 \%$ (from 7.21 to $2.62 \mathrm{~mm} \mathrm{~h}^{-1}$ ) in the $Z$ bias_LGC. In correlation coefficient, the accuracy of the RAR system was also improved by about $10.7 \%$ (from 0.84 to 0.93 ) in $Z$ bias_MFBC and $11.7 \%$ (from 084 to 0.94 ) in $Z$ bias_LGC. It is proved that the accuracy of rainfall estimates in the RAR system was improved by the $Z$ bias with rainfall-bias correction methods more than only the $Z$ bias. Especially, among the rainfall-bias correction methods, the $Z$ bias_LGC is superior to others. The reason is that although the same rainfall-bias was applied to the overall application basin in the MFBC method, the different rainfall biases were applied to each rainfall amount by radar pixel in the LGC method. In Table 4b, although correlation coefficients in the $Z$ bias 25 correction were similar to all rainfall types, typhoon cases had the lowest accuracy in RMSE. As a result of the $Z$ bias_MFBC, correlation coefficients in all types were improved when compared with $Z$ bias. While the accuracy of the $Z$ bias_MFBC in RMSE improved over the $Z$ bias except for in Changma front cases, results of typhoon 
cases were inferior to others as always. Results of the $Z$ bias_LGC showed that the accuracy of rainfall estimates for all types in RMSE and correlation coefficients was superior to the $Z$ bias and, especially, outcomes in typhoon cases were vastly superior to the others. Figure 9 has explained that the RMSEs of the $Z$ bias_LGC of all cases

5 were outstanding in Fig. 9a and while correlation coefficients of the $Z$ bias_MFBC were not much different to the $Z$ bias_LGC on average, only the $Z$ bias correction results were generally lower in Fig. $9 \mathrm{~b}$.

Figure 10 shows rainfall estimate images of AWS, $Z$ bias, $Z$ bias_MFBC, and $Z$ bias_LGC in Case 12 (at 15:00 LST on 10 August in 2012) and Case 18 (at 11:00 LST

10 on 30 August in 2012). In Fig. 10a in Case 12, the maximum rainfall amount in AWSs was $48 . \mathrm{mm} \mathrm{h}^{-1}$ and the black arrows indicate the strongest rainfall fields. Figure 10b shows that since the displayed rainfall regions were similar to AWSs, rainfall amounts were underestimated in the whole area. As an image of the $Z$ bias_MFBC in Fig. 10c, rainfall amounts in a black circle were closer to AWSs than Fig. 10b. Especially, the im-

15 age of the $Z$ bias_LGC is similar to AWSs and rainfall estimates which ranged from 40 to $50 \mathrm{~mm} \mathrm{~h}^{-1}$ in the regions, indicated by black arrows in a black circle were similar to AWSs. In Fig. 11a in case 18, the maximum rainfall amount in AWSs was 54. $\mathrm{mm} \mathrm{h}^{-1}$ and the rainfall fields indicated by black arrows were stronger than the others. Particularly, the rainfall zones (the black dash line) from the southwest to the northeast 20 occurred due to the direct effects of the typhoon Tembin along its track (the purple line). Figure $11 \mathrm{~b}$ shows rainfall amounts in only the $Z$ bias were much underestimated in whole area. By contrast, in Fig. 11c for the $Z$ bias_MFBC, the maximum rainfall estimates in a region (a) which was located in the southeast of the Tembin and in rainfall zones from the southwest to the northeast (region (b)) were much improved.

25 However, rainfall estimates in region (a) were a little underestimated and the region (b) had slightly strong rainfall amounts. In Fig. $11 \mathrm{~d}$, since rainfall estimates in region (c) were stronger than for region (a) and the region (d) had lighter rainfall amounts than the region (b), an image of rainfall estimates in the $Z$ bias_LGC was coterminous with AWSs. It is proved that the accuracy of the rainfall estimates in the RAR system with

4025

the rainfall-bias correction is improved compare to using only the $Z$ bias correction. Especially, the $Z$ bias_LGC is superior to the others.

\section{Conclusions}

This paper focuses on correcting the reflectivity measurement bias ( $Z$ bias) which in5 cludes temporal and spatial sampling bias, ground and sea clutter, beam-blockage and attenuation, electrical calibration, and quantification of reflectivity bias and the QPE model bias (rainfall-bias) which includes bias due to the parameters of $Z-R$ relationship, parameters of the QPE model, and the QPE model structure to improve radar rainfall estimates. The reference radar, Bislsan $S$ band dual-polarimetric radar, which

10 was self-calibrated with the self-consistency constraint method using the relationship between $Z$ and $K_{\mathrm{DP}}$ was utilized to calculate the $Z$ biases of all target radar sites and $Z$ biases were applied to the QPE model, RAR system. The MFBC and LGC methods which correct rainfall-biases have also been applied to the RAR system to improve the accuracy of radar rainfall estimates.

15 As a result of the $Z$ bias correction in 18 summer season cases, the accuracy of rainfall estimates improved in the RMSE and correlation coefficient which ranged from $7.37 \mathrm{~mm} \mathrm{~h}^{-1}$ and 0.83 and $7.21 \mathrm{~mm} \mathrm{~h}^{-1}$ and 0.84 on average, respectively and, for rainfall types, the accuracy of rainfall estimates in Changma front and local torrential cases was slightly improved or similar to results without the application of $Z$ bias. Espe20 cially, the accuracy of typhoon cases was worse than existing results (from 9.08 to $11.04 \mathrm{~mm} \mathrm{~h}^{-1}$ ). The reason is that the application of $Z$ biases to each radar site in the RAR system has increased the rainfall estimates for the whole country. The accuracy of Changma front cases, which occur nationwide, was improved. However, because cases of torrential rainfalls and typhoon have occurred locally, the accuracy of these

25 cases was worsened. In comparison with rainfall images, rainfall estimates with the $Z$ bias correction have been established to be stronger to existing image. 
Since the rainfall estimates in the RAR system has been improved by the $Z$ bias correction, the QPE model bias (rainfall-bias) correction was conducted after the $Z$ bias correction. For results of the rainfall-bias correction methods, the accuracy of rainfall estimates with the $Z$ bias_MFBC was improved by about $7.4 \%$ in RMSE and $10.7 \%$

5 in correlation coefficient in comparison with only the $Z$ bias, respectively, and the accuracy of the $Z$ bias_LGC was especially superior to the others $(63.7 \%$ in RMSE and $11.7 \%$ in correlation coefficient). The reason is that although the same rainfall-bias was applied to the all over area in the MFBC method, the different rainfall biases were applied to each rainfall amount by radar pixel in the LGC method. For rainfall types,

10 results of the $Z$ bias_LGC showed that the accuracy of rainfall estimates for all types in RMSE and correlation coefficient was much improved over only the $Z$ bias and, especially, outcomes in typhoon cases were vastly superior to the others. In comparison of rainfall images, rainfall estimates with the $Z$ bias_LGC were determined to closer to AWSs in the cases of Changma fronts and the typhoon, Tembin.

Therefore, in this paper, it is proved that the accuracy of the rainfall estimates in the RAR system, to which the $Z$ bias correction and rainfall-bias correction method (MFBC and LGC) were applied, has been improved. These bias correction methods proposed by this paper are able to contribute to the real-time QPE model, the RAR system, in work-site operation and to fundamental bias correction research. However, this paper

20 has dealt with the bias corrections in a few parts in a series of a procedure. Since radar rainfall estimates are still based on a series of assumptions, more research on numerous systematic biases, also including natural biases, should be undertaken the performance of the calculation of radar-based rainfall estimates.

Acknowledgements. This research is supported by "Development and application of Cross gov25 ernmental dual-pol radar harmonization (WRC-2013-A-1)" project of the Weather Radar Center, Korea Meteorological Administration in 2014 and Deajin University.

4027

\section{References}

Austin, P. M.: Relation between measured radar reflectivity and surface rainfall, Mon. Weather Rev., 115, 1053-1070, 1987.

Campos, E. and Zawadzki, I.: Instrumental uncertainties in $Z-R$ relations, J. Appl. Meteorol., 39, 1088-1102, 2000.

Chumchean, S., Sharma, A., and Seed, A.: An integrated approach to error correction for realtime radar-rainfall estimation, J. Atmos. Ocean. Tech., 23, 67-79, 2006.

Ehret, U.: Rainfall and Flood Nowcasting in Small Catchments Using Weather Radar, Ph.D. thesis, University of Stuttgart, 2002.

10 Germann, U., Galli, G., Boscacci, M., and Bolliger, M.: Radar precipitation measurement in a mountainous region, Q. J. Roy. Meteor. Soc., 132, 1669-1692, 2006.

Huff, F. A.: Sampling errors in measurement of mean precipitation, J. Appl. Meteorol., 9, 35-44, 1970.

Jordan, P., Seed, A., Austin, G.: Sampling errors in radar estimates of rainfall, J. Geophys. Res.-Atmos., 105, 2247-2257, 2000.

Kim, D.-S., Kang, M.-Y., Lee, D.-I., Kim, J.-H., Choi, B.-C., and Kim, K. E.: Reflectivity $Z$ and differencial reflectivity ZDR correction for polarimetric radar rainfall measurement, in: Proceeding of the Spring Meeting of Korean Meteorological Society, 130-131, 2006.

Korea Meteorological Administration: Development of Collaboration Management System For Radar Data (I), Weather Radar Center, 2011.

Korea Meteorological Administration: Development of Radar-Based Multi-Sensors Quantitative Precipitation Estimation Technique Report, Weather Radar Center, 2012a.

Korea Meteorological Administration: Radar Rainfall Estimation Comparison and Verification Joint Experiment Report, Weather Radar Center and Meteorological Advancement Council, Korea Meteorological Administration, 2012b.

Krajewski, W. F. and Smith, J.: Radar hydrology: rainfall estimation, Adv. Water Resour., 25, 1387-1394, 2002.

Lee, G. W.: Sources of errors in rainfall measurements by polarimetric radar: variability of drop size distributions, observational noise, and variation of relationships between $R$ and polarimetric parameters, J. Atmos. Ocean. Tech., 23, 1005-1028, 2006.

Marshall, J. S. and Palmer, W. M.: The distribution of raindrops with size, J. Meteorol., 5, 165$166,1948$. 
McMillan, H., Jackson, B., Clark, M., Kavetski, D., and Woods, R.: Rainfall uncertainty in hydrological modeling: an evaluation of multiplicative error models, J. Hydrol., 400, 83-94, 2011.

Moulin, L., Gaume, E., and Obled, C.: Uncertainties on mean areal precipitation: assessment and impact on streamflow simulations, Hydrol. Earth Syst. Sci., 13, 99-114, doi:10.5194/hess-13-99-2009, 2009.

Morin, E. and Gabella, M.: Radar-based quantitative precipitation estimation over Mediterranean and dry climate regimes, J. Geophys. Res., 112, D20108, doi:10.10029/2006JD008206, 2007.

Oh, H.-M., Ha, K.-J., Kim, K.-E., and Bae, D.-H.: Precipitation rate combined with the use of optimal weighting of radar and rain gauge data, Atmosphere, Korean Meteorological Society, 13, 316-317, 2003.

Rosenfeld, D., Wolff, D. B., and Atlas, D.: General probability-matched relations between radar reflectivity and rain rate, J. Appl. Meteorol., 32, 50-72, 1993.

Sinclair, S. and Pegram, G.: Combining radar and rain gauge rainfall estimates using conditional merging, Atmos. Sci. Lett., 6, 19-22, 2005.

Villarini, G.: Empirically-based modeling of radar-rainfall uncertainties, Ph. D. thesis, The University of lowa, 321, 2008.

Villarini, G. and Krajewski, W. F.: Empirically-based modeling of spatial sampling uncertainties associated with rainfall measurements by rain gauges, Adv. Water Resour., 31, 1015-1023, 2008.

Villarini, G. and Krajewski, W. F.: Sensitivity studies of the models of radar-rainfall uncertainties, J. Appl. Meteorol. Clim., 49, 288-309, 2010.

Villarini, G., Mandapaka, P. V., Krajewski, W. F., and Moore, R. J.: Rainfall and sampling errors: a rain gauge perspective, J. Geophys. Res., 113, D11102, doi:10.1029/2007JD009214, 2008.

Weather Radar Center: Development of Integrated Quality Control Algorithm For Korean Radar Data, Korea Meteorological Administration, 2013.

Wilson, J. W. and Brandes, E. A.: Radar measurement of rainfall, B. Am. Meteorol. Soc., 60, 1048-1058, 1979.

30 Woodley, W., Olsen, A., Herndon, A., and Wiggert, V.: Comparison of gage and radar methods of convective rain measurement, J. Appl. Meteorol., 14, 909-928, 1975.

Yoo, C., Kim, J., Yoon, J., Park, C., Park, C., and Jun, C.: Use of the Kalman filter for the correction of mean-field bias of radar rainfall, The 5th Korea-Japan-China Joint Conference on Meteorology, Busan, Korea, 2011.

Zhang, J., Howard, K., Langston, C., Vasiloff, S., Kaney, B., Arthur, A., Cooten, V. C., Kelle-

5 her, K., Kitzmiller, D., Ding, F., Seo, D.-J., Wells, E., and Dempsey, C.: National mosaic and multi-sensor QPE(NMQ) system: desscription, results, and future plans, B. Am. Meteorol. Soc., 92, 1321-1338, 2011.

Zhang, Y., Adams, T., and Bonta, J. V.: Subpixelscale rainfall variability and the effects on the separation of radar and gauge rainfall errors, J. Hydrometeorol., 8, 1348-1363, 2007. 
Table 1a. Summary of the radars and rainfall cases. Summary of the radars and rainfall data used for calculating observational biases.

\begin{tabular}{|c|c|}
\hline Items & Details \\
\hline Reference radar & $\begin{array}{l}\text { Bislsan } S \text { band dual-polarization radar } \\
\text { (maximum observation range: } 150 \mathrm{~km} \text {; gate size: } 0.125 \mathrm{~km} \text {; elevation: } 6 \text { angles; update: } \\
\text { every } 2.5 \mathrm{~min} \text { interval) }\end{array}$ \\
\hline Target radar & $\begin{array}{l}11 \text { single-polarization radars operated by the Korea Meteorological Administration: } \\
\text { Baegnyeondo (BRI, } S \text { band), Kwanaksan (KWK, } S \text { band), Oseonsan (KSN, } S \text { band), Jindo } \\
\text { (JNI, } S \text { band), Gosan (GSN, } S \text { band), Seongsan (SSP, } S \text { band), Gudeoksan (PSN, } S \\
\text { band), Myeonbongsan (MYN, } C \text { band), Gangneung (GNG, } S \text { band), Gwnagdeoksan (GDK, } \\
S \text { band), Incheon (IIA, } C \text { band) }\end{array}$ \\
\hline Calibration data & Rainfall cases from 1 Jun to 31 Aug in 2012 \\
\hline
\end{tabular}

4031

Table 1b. Summary of the radars and rainfall cases. Rainfall cases used for verification of the observational and model bias correction.

\begin{tabular}{lll}
\hline Items & Period (LST) & Sources \\
\hline Case 1 & 8 Jun 2012 06:00-8 Jun 2012 19:00 & Local torrential rainfalls \\
Case 2 & 15 Jun 2012 05:00-16 Jun 2012 04:00 & Changma front \\
Case 3 & 18 Jun 2012 00:00-19 Jun 2012 13:00 & Changma front \\
Case 4 & 23 Jun 2012 13:00-24 Jun 2012 19:00 & Local torrential rainfalls \\
Case 5 & 29 Jun 2012 08:00-1 Jul 2012 01:00 & Changma front \\
Case 6 & 5 Jul 2012 04:00-7 Jul 2012 02:00 & Changma front \\
Case 7 & 10 Jul 2012 10:00-11 Jul 2012 19:00 & Changma front \\
Case 8 & 12 Jul 2012 23:30-13 Jul 2012 07:30 & Changma front \\
Case 9 & 14 Jul 2012 08:00-15 Jul 2012 15:00 & Changma front \\
Case 10 & 16 Jul 2012 23:00-17 Jul 2012 22:00 & Changma front \\
Case 11 & 18 Jul 2012 14:00-19 Jul 2012 13:00 & Typhoon \\
Case 12 & 10 Aug 2012 03:00-10 Aug 2012 22:00 & Local torrential rainfalls \\
Case 13 & 12 Aug 2012 05:00-13 Aug 2012 15:00 & Local torrential rainfalls \\
Case 14 & 14 Aug 2012 17:00-16 Aug 2012 23:00 & Local torrential rainfalls \\
Case 15 & 19 Aug 2012 16:00-22 Aug 2012 21:00 & Local torrential rainfalls \\
Case 16 & 22 Aug 2012 22:00-25 Aug 2012 11:00 & Local torrential rainfalls \\
Case 17 & 27 Aug 2012 13:00-28 Aug 2012 18:00 & Changma front and Typhoon \\
Case 18 & 29 Aug 2012 15:00-30 Aug 2012 23:00 & Typhoon \\
\hline
\end{tabular}


Table 2. Radar pairs for estimating the $Z$ bias of each radar site.

\begin{tabular}{llll}
\hline Reference radar & Target radar & Reference radar & Target radar \\
\hline BSL & KSN, PSN, MYN & IIA & BRI \\
KSN & JNI & KSN & KWK \\
JNI & GSN, SSP & KWK & GDK \\
KWK & IIA & GDK & GNG \\
\hline
\end{tabular}

Table 3. Reflectivity bias for each radar site.

\begin{tabular}{llll}
\hline Radar site & Reflectivity bias $(\mathrm{dB})$ & Radar site & Reflectivity bias $(\mathrm{dB})$ \\
\hline BRI & $-7.87^{*}$ & $\mathrm{JNI}$ & -1.16 \\
GDK & -4.29 & $\mathrm{KSN}$ & -4.87 \\
GSN & -3.99 & $\mathrm{KWK}$ & -5.15 \\
GNG & -4.77 & $\mathrm{MYN}$ & -5.63 \\
IIA & -5.19 & $\mathrm{PSN}$ & -2.28 \\
SSP & -4.50 & & \\
\hline
\end{tabular}

${ }^{*}$ Average reflectivity bias during the calibration period. 
Table 4a. Application results of the QPE model bias correction methods. Total average.

\begin{tabular}{llll}
\hline Method & MAE $\left(\mathrm{mm} \mathrm{h}^{-1}\right)$ & RMSE $\left(\mathrm{mm} \mathrm{h}^{-1}\right)$ & Correlation coefficient \\
\hline$Z$ bias & 3.65 & 7.21 & 0.84 \\
$Z$ bias_MFBC & 3.37 & $6.68\left(7.4 \%^{*}\right)$ & $0.93(10.7 \%)$ \\
$Z$ bias_LGC & 2.19 & $2.62(63.7 \%)$ & $0.94(11.7 \%)$ \\
\hline
\end{tabular}

${ }^{*}$ Represents the change ratio related to the OBC method in RMSE and correlation coefficient.

Table 4b. Application results of the QPE model bias correction methods. Average for each rainfall type.

\begin{tabular}{llll}
\hline Method & Types & Averaged RMSE $\left(\mathrm{mm} \mathrm{h}^{-1}\right)$ & $\begin{array}{l}\text { Averaged correlation } \\
\text { coefficient }\end{array}$ \\
\hline$Z$ bias & Changma front & 5.64 & 0.87 \\
& Local torrential rainfall & 7.36 & 0.81 \\
& Typhoon & 11.04 & 0.83 \\
\hline$Z$ bias_MFBC & Changma front & 5.75 & 0.93 \\
& Local torrential rainfall & 6.74 & 0.95 \\
& Typhoon & 9.00 & 0.86 \\
\hline$Z$ bias_LGC & Changma front & 2.49 & 0.95 \\
& Local torrential rainfall & 2.69 & 0.94 \\
& Typhoon & 2.81 & 0.93 \\
\hline
\end{tabular}




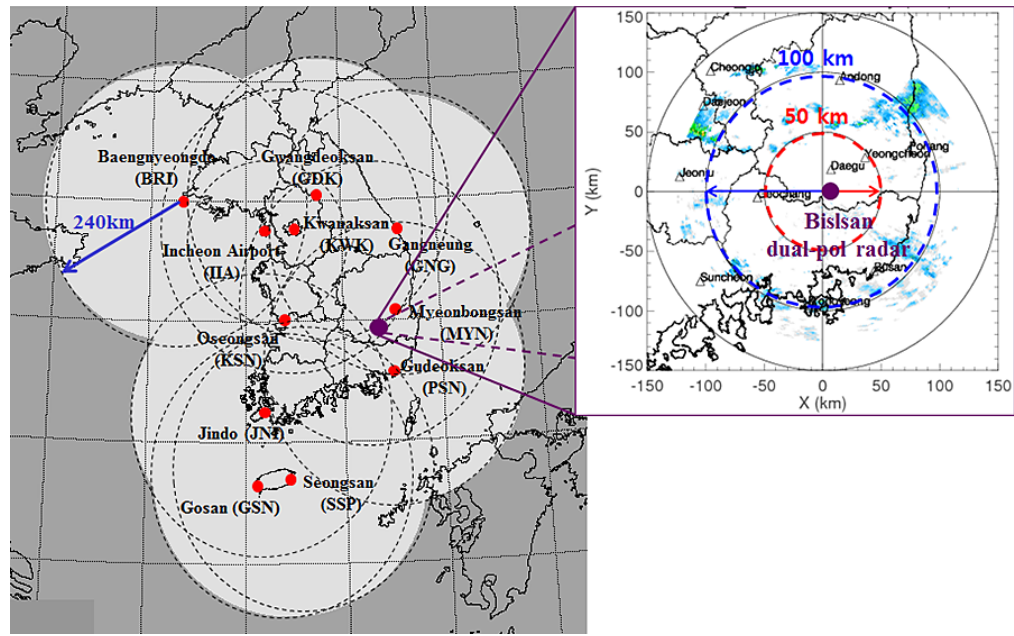

Figure 1. Location of 11 single-polarization radars and the Bislsan $S$ band dual-polarization radar and their observation ranges.

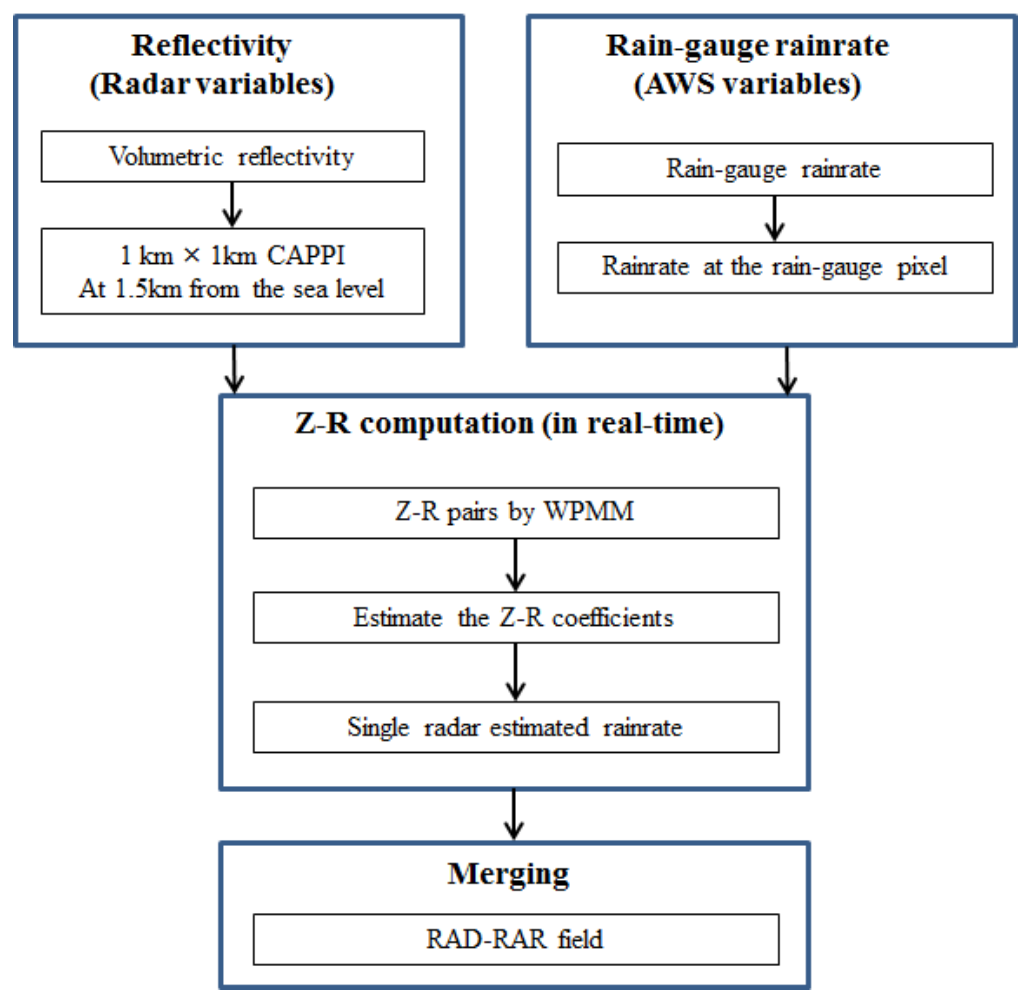

Figure 2. Flowchart of the Radar-AWS Rainrate calculation system. 


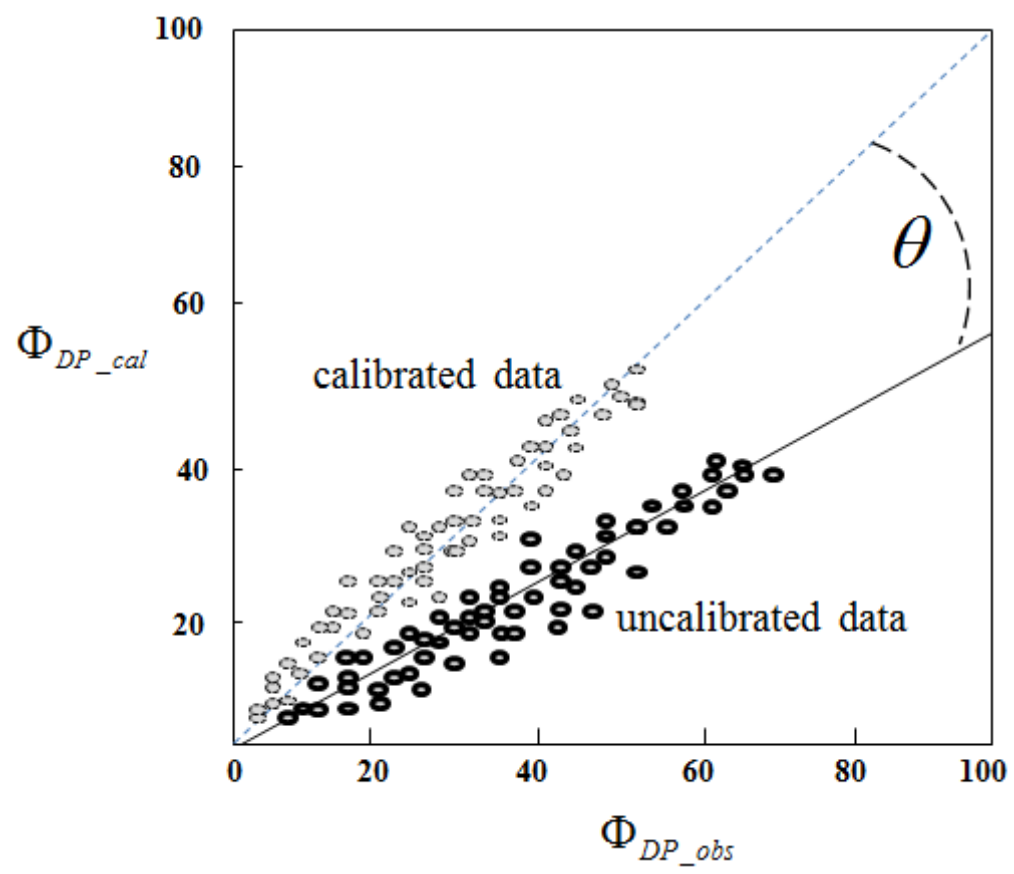

Figure 3. Example for the procedure of the self-consistency constraint: calculation of $\tan \theta$ using Eq. (3).

4039

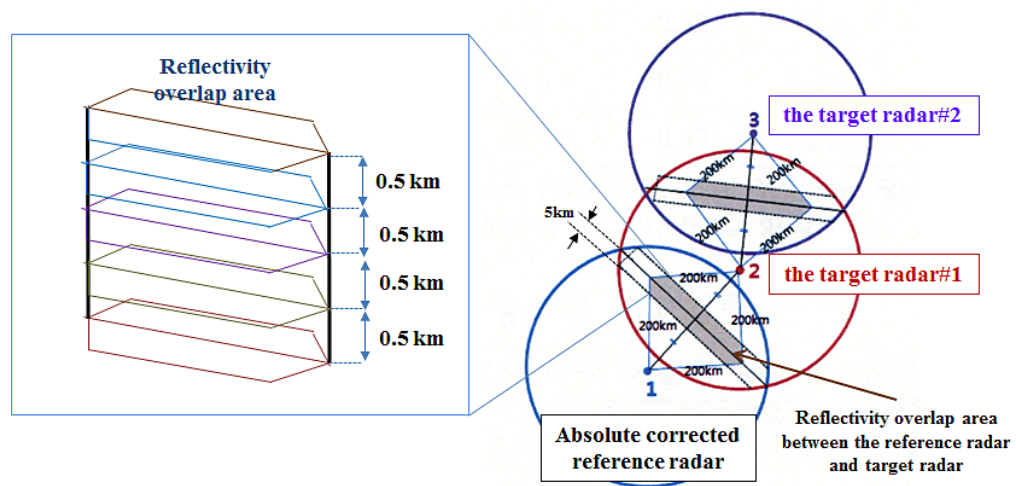

Figure 4. The concept of calculating $Z$-bias for the target radar according to the reference radar reflectivity (Korea Meteorological Administration, 2011). 


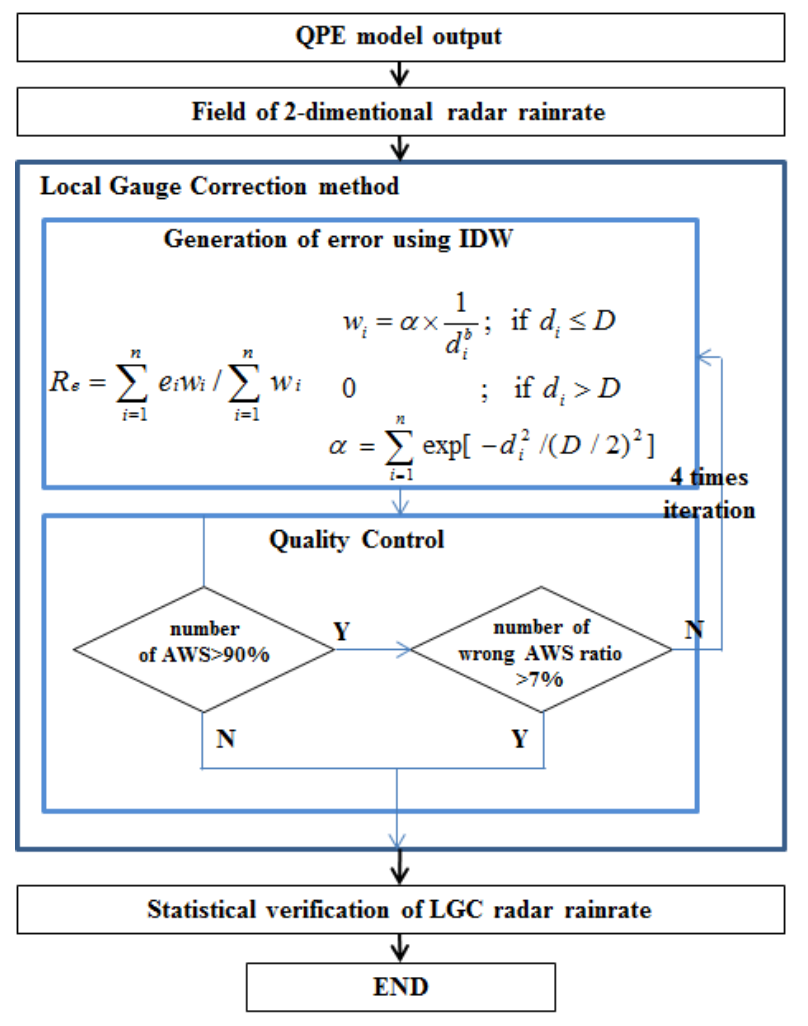

Figure 5. Flowchart of the Local Gauge Correction method.

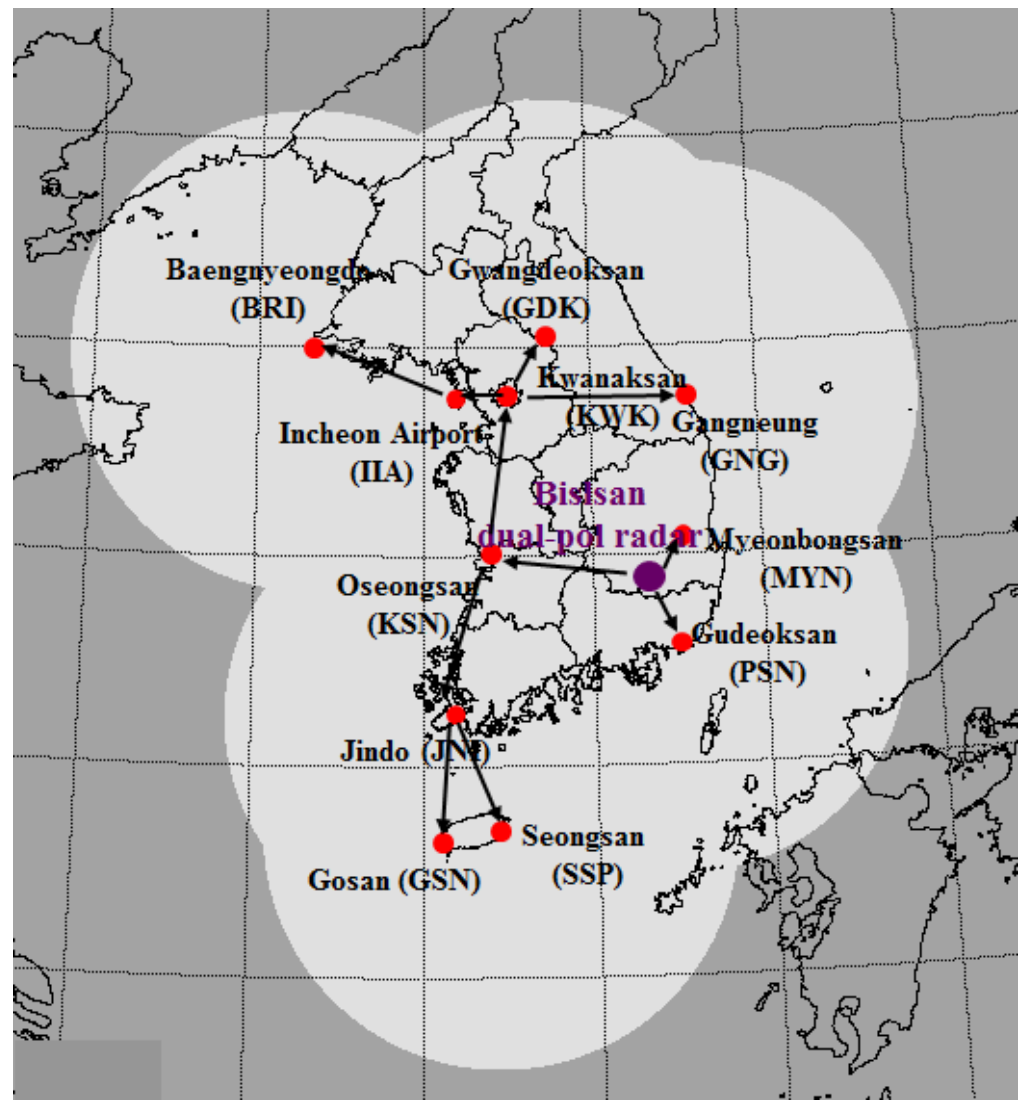

Figure 6. Sequence of the reflectivity bias estimation for each radar site. 

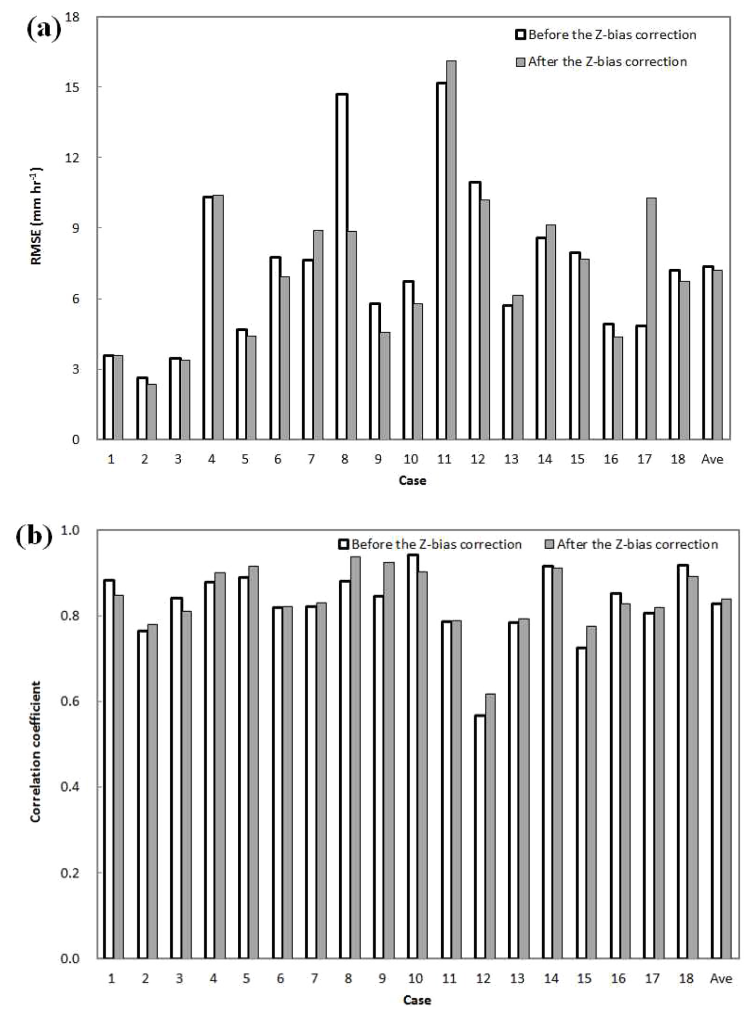

Figure 7. Comparison of the accuracy of rainfall estimates for each rainfall case before and after the $Z$ bias correction: (a) RMSE; (b) correlation coefficient.

4043

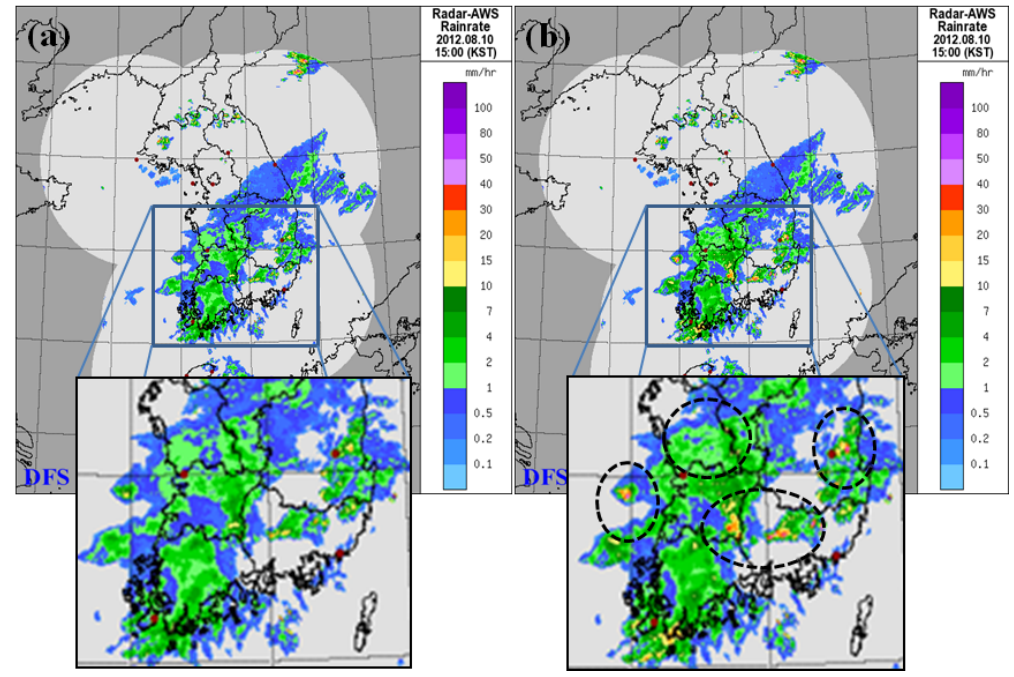

Figure 8. Comparison of rainfall estimate images in the RAR system before and after the $Z$ bias correction in Case 12 (at 15:00 LST on 10 August in 2012): (a) before the $Z$ bias correction; (b) After the $Z$ bias correction. 

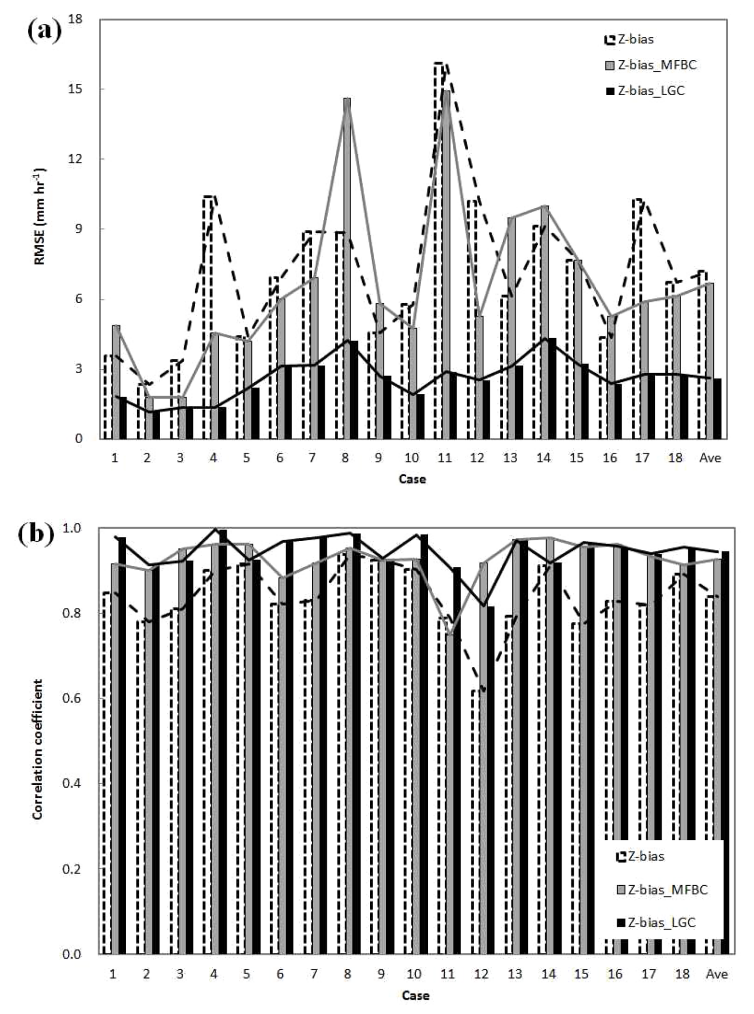

Figure 9. Comparison of the rainfall estimation accuracy for each rainfall in the $Z$ bias, $Z$ bias_MFBC, and $Z$ bias_LGC methods: (a) RMSE; (b) correlation coefficient.
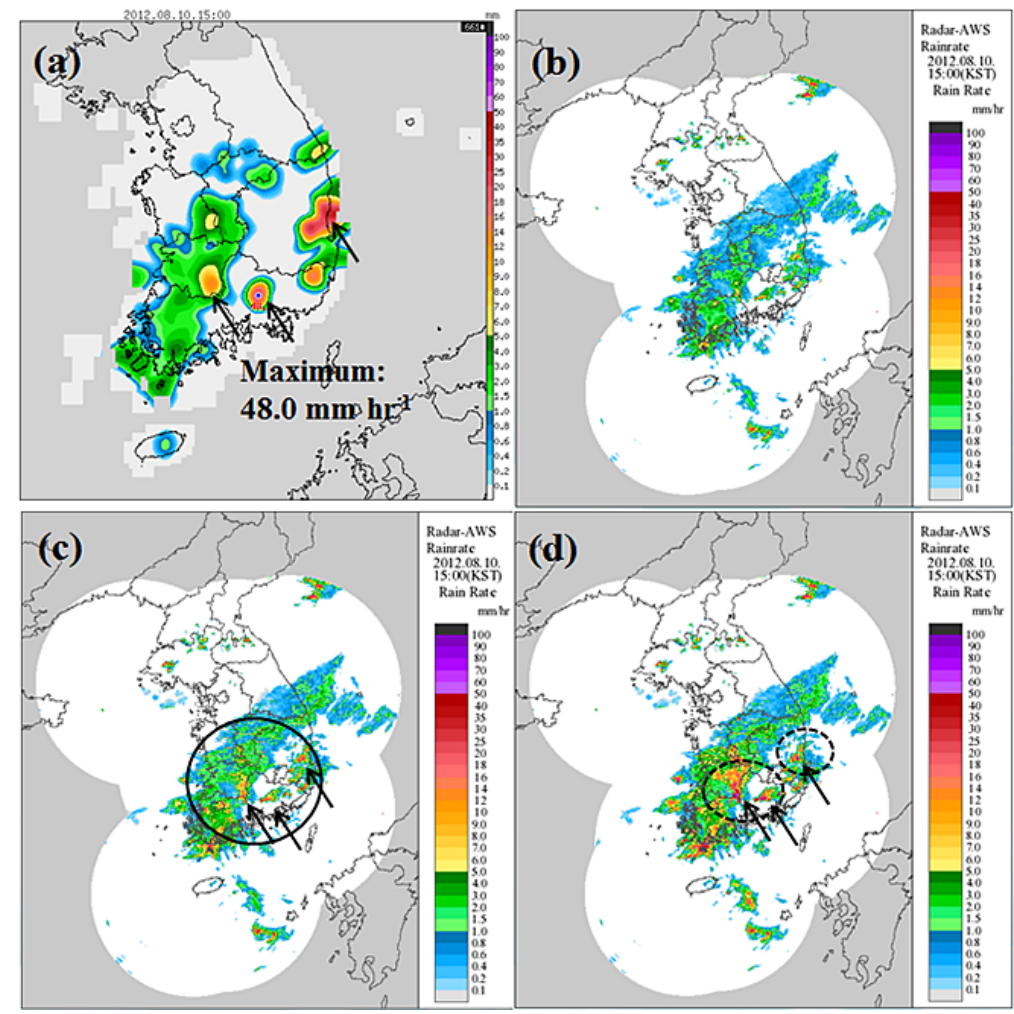

Figure 10. Comparison of the rainfall images between the AWS and Model Bias Correction method results in case 12 (at 15:00 LST on 10 August in 2012): (a) the AWS; (b) the OBC method; (c) the OBC_MFBC method; (d) the OBC_LGC method. 


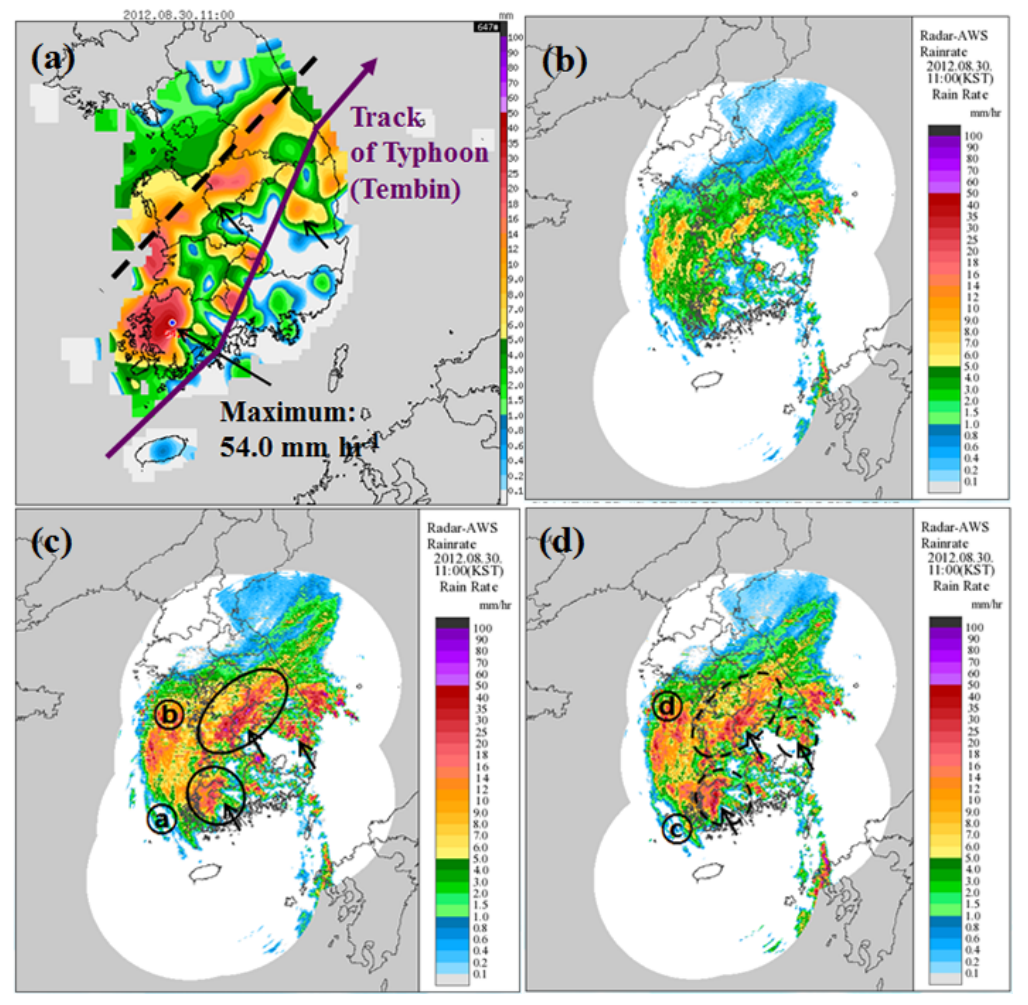

Figure 11. Comparison of the rainfall images between the AWS and Model Bias Correction method results in case 18 (at 11:00 LST on 30 August in 2012): (a) the AWS; (b) the OBC method; (c) the OBC_MFBC method; (d) the OBC_LGC method. 